Philosophie ANTIQUE

\section{Philosophie antique}

Problèmes, Renaissances, Usages

$10 \mid 2010$

Philosophie et mathématiques

\title{
Lambros COULOUBARITSIS, La pensée de Parménide
}

(en appendice traduction du Poème), Éditions Ousia, Bruxelles, 2008, 570 p. ISBN 978-2-87060-142-6

Dario Zucchello

\section{OpenEdition}

\section{Journals}

Edizione digitale

URL: https://journals.openedition.org/philosant/2370

DOI: 10.4000/philosant.2370

ISSN: 2648-2789

Editore

Éditions Vrin

\section{Edizione cartacea}

Data di pubblicazione: 30 octobre 2010

Paginazione: $278-281$

ISBN: 978-2-7574-0179-8

ISSN: 1634-4561

\section{Notizia bibliografica digitale}

Dario Zucchello, «Lambros couloubaritsis, La pensée de Parménide », Philosophie antique [En ligne], 10 | 2010, mis en ligne le 11 juillet 2019, consulté le 05 décembre 2022. URL : http:// journals.openedition.org/philosant/2370; DOI : https://doi.org/10.4000/philosant.2370

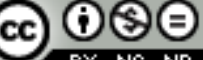

Creative Commons - Attribution - Pas d'Utilisation Commerciale - Pas de Modification 4.0 International - CC BY-NC-ND 4.0

https://creativecommons.org/licenses/by-nc-nd/4.0/ 
sui «Presocratici come filosofi» (nella raccolta di Laks e Louguet). Avrebbero potuto suggerire una ricostruzione più equilibrata della fisionomia del poema.

Dario ZUCCHELLO

Lambros Couloubaritsis, La pensée de Parménide (en appendice traduction du Poème), Éditions Ousia, Bruxelles, 2008, 570 p. ISBN 978-2-87060-142-6

Nel caso di riedizione di opera ormai classica, normalmente le ragioni che hanno spinto l'autore a riproporne o rivederne l'impianto sono proposte nella nuova prefazione o in introduzione: non è facile, invece, imbattersi - anche all'interno del corpo del testo vero e proprio - nella esplicita confessione del travaglio teorico all'origine del ripensamento, della rielaborazione e della revisione delle proprie posizioni interpretative. È quello che accade affrontando la lettura della recente terza rivisitazione di Mythe et Philosophie chez Parménide di Lambros Couloubaritsis, così ampia da giustificare una titolazione più comprensiva e impegnativa: La pensée de Parménide.

Quasi alla conclusione del Capitolo II (Le Proème comme producteur de chemins), nel contesto di un confronto articolato e puntuale con le interpretazioni più recenti circa natura e funzione del primo segmento testuale del poema, l'autore, interrogandosi sul rischio che la lettura puramente metaforica delle figure di Dike, Ananke, Moira (e Aletheia) possa comportare la riduzione della nozione stessa di Eon (di cui quei numi sono posti a garanzia) a «mera finzione», osserva: "Cette question qui nous trouble depuis quelques années, et qui a suscité en nous le besoin de repenser la question du mythe, s'installe [...] comme une énigme, une aporie qu'on ne peut plus laisser dans l'ombre où elle se plaît à résider, inviolable comme l'Eon parménidien» (p. 191). Insomma, il nodo che Couloubaritsis ancora individua come essenziale per l'ermeneusi del poema è lo stesso all'origine della prima ricerca e di altri suoi importanti contributi parmenidei (Les multiples chemins de Parménide, in Études sur Parménide, sous la direction de P. Aubenque, Tome II: Problèmes d'interprétation, Vrin, Paris 1987, p. 25-43): il nesso tra gli echi del mito, a partire dal Proemio, e la struttura teorica dell'opera. L'attenzione a questo tema si è poi tradotta nello sviluppo di due fondamentali (e complementari) direttrici d'indagine: la "deontologizzazione" della interpretazione del poema e il recupero della centralità della sezione sulla Doxa come tentativo di fondazione di una nuova «fisica».

Rispetto alle edizioni precedenti colpiscono positivamente due elementi di fondo della ricerca: l'ampiezza e la profondità della prospettiva, in altre parole la pluralità di punti di vista e lo spettro culturale considerati, insieme allo spessore della dimensione diacronica, che tende a collocare il pensiero di Parmenide saldamente entro la tradizione del pensiero e della letteratura arcaica, proiettandolo verso Platone e Aristotele. Risalta allora significativamente l'assenza totale, nell'aggiornamento del dibattito e nella costruzione della linea di lettura, del riferimento ai contributi di Peter Kingsley - Ancient Philosophy, Mystery and Magic. Empedocles and the Pythagorean Tradition (1995), In the Dark Places of Wisdom (1999), Reality (2003) - che negli ultimi due decenni hanno tracciato una interessante alternativa, radicando e retroiettando piuttosto l'Eleate all'interno di un passato 
di originali esperienze culturali (sciamanesimo), dalle sponde orientali e occidentali del mondo greco (da Focea alla Magna Grecia, alla Sicilia), con conseguente profondo ripensamento dell'intenzione del poema (guidare a una esperienza "vissuta" e ineffabile di comprensione della realtà). Tanto più significativo se consideriamo che Couloubaritsis discute a più riprese la recente posizione interpretativa di Chiara Robbiano (Becoming Being. On Parmenides' Transformative Philosophy, 2006), che a quei contributi e a quella possibilità di lettura in chiave "trasformativa" dei frammenti parmenidei esplicitamente allude. Una prospettiva per altro rilanciata - in termini più marcatamente pedagogici e non senza presa di distanze dalla lettura "sciamanica" di Kingsley - anche dalla compianta Martina Stemich (Parmenides' Einübung in die Seinserkenntnis, 2008).

Contro la tendenza a valorizzare nel poema la rappresentazione di un processo di conversione spirituale, dunque, la proposta dell'autore, pur avvertita della esigenza di desaturarne le presunte ambizioni ontologiche, è decisamente teoretica. Il Peri physeos di Parmenide prefigurerebbe, sin dai contenuti narrativi del mito proemiale («mythe de l'origine et de la genèse du savoir», p. 526), una peculiare aspirazione al sapere (philo-sophia appunto), di cui sarebbero anticipati allegoricamente i dati ultimi, quelli cui condurranno i percorsi del sapere. Una enquête, dunque, snodantesi per multiples chemins: una quête du savoir dovrà, infatti, misurarsi anche con le formes du non-savoir (p. 54-55). L'uso del mito supplisce l'assenza di una esplicita rivendicazione "filosofica" nel testo: è all'interno della sua costruzione che sono legittimati tanto l'Eon quanto il pensiero, così come il complesso sforzo per pensare - secondo condizioni pertinenti - il mondo del divenire, «destinazione finale» del cammino intrapreso sotto la direzione divina. L'approccio doxastique della seconda parte del poema avrebbe in questo senso inaugurato una nuova physique du mélange (p. 20), ripresa e diversamente modulata da Empedocle, Anassagora e atomisti: ribadendo la propria tesi, Couloubaritsis si ritrova in sintonia con una linea di lettura costruttiva della Doxa, che annovera importanti adesioni tra gli interpreti più recenti (Curd, Thanassas, Graham, Lesher).

Alla "deontologizzazione" del poema contribuiscono soprattutto due elementi caratteristici della lezione dell'autore: la già segnalata valorizzazione dei multiples chemins (punto di forza della decennale attività interpretativa di Couloubaritsis sul testo parmenideo) e la nuova esegesi dell'Eon eleatico.

Così, riprendendo da Mourelatos la distinzione tra motivo, tema e simbolizzazione, lo studioso individua nello «schema» del cammino (bodos) ovvero pista (patos) o via (keleuthos) - il vero nucleo propulsivo dell'opera (cui ricondurre anche il motivo del viaggio), che organizza in narrazione unitaria e sistematica una costellazione di elementi significativi per la cultura greca arcaica: $i$ tre segmenti fondamentali (proemio, discorso sull'Eon e discorso doxastique) manifesterebbero l'impianto fondamentalmente ancora catalogico del discorso parmenideo, e dunque la sua collocazione interna al logos arcaico e alle sue dinamiche, che Couloubaritsis preferisce al consunto stereotipo di un passaggio dal mythos al logos. Quello schema, unitamente alla funzione della guida divina e delle sue proiezioni (Dike, Ananke, Moira), si impone non solo come condizione di continuità, ma essenzialmente come principio "trascendentale". Introdotto nel 
racconto proemiale, esso offre infatti il contesto entro cui Eon e Doxa assumono il loro senso e il loro statuto: appunto in quanto appartenenti a uno specifico «cammino» tra gli altri possibili. Indicativo, in particolare, a dispetto delle tradizionali riduzioni ontologiche, il rilevamento della «eccedenza», del «trascendimento» (débordement) dello schema rispetto all'Eon, che emerge in fondo "solo" come l'unico percorso in grado di condurre a destinazione nella ricerca del sapere.

Proprio nell'ottica dello «schema del cammino è possibile avvertire come l'emergere del logos manifesti in realtà una transmutation del mythos: è solo a partire dal mito dei molteplici cammini che si dipana il «discorso molteplice» della Dea, che nel corso della narrazione verterà sull'essere, sul non-essere e sulle differenti doxai. Si tratta, secondo Couloubaritsis, della conditio sine qua non per emancipare il poema dalle prospettive ermeneutiche forzate su di esso: l'unica possibilità per superare l'apparente contraddizione della presenza di figure mitiche (Dike, Ananke, Moira) che eccedono (garantendolo) ciò che pretende - di fronte al pensiero - dominare senza eccezione: l'Eon.

Il proemio inscena l'incontro del giovane eroe con la Dea, cui spetta il compito di rivelare il tutto del sapere e del non-sapere: la prima parte del suo discorso fa irrompere sulla scena il pensiero, di cui l'Eon, considerato nella modalità temporale appropriata (l'«ora»), è condizione; la seconda parte, invece, porta a compimento la comunicazione (e il poema) con lo studio del divenire mediante «des références pertinentes» e la temporalità propria del pensiero arcaico (ta te eonta, ta pro eonta, ta essomena) (p. 102). Nei due momenti del discorso divino si riflette dunque, profondamente rielaborata, la tensione implicita tra la dimensione temporale delle cose in divenire e quella peculiare alla concezione arcaica delle divinità («cose che sono sempre nel presente», aien eonton). Donde la decisione di Couloubaritsis - coerente con l'intenzione di "deontologizzare" la lettura del poema - di rompere con la consueta resa del greco eon (essere, ente), a favore di un improbabile ma efficace «ce qui est dans le présent». Una scelta che ne rafforza indubbiamente la linea interpretativa rispetto al testo parmenideo, nei cui segmenti sarebbero evidenziati da un lato la ripresa di elementi e motivi dell'ambiente culturale, dall'altro la loro tematizzazione originale, nella messa in scena della quête du savoir (p. 203).

La chiave dei multiples chemins, tradotta in pluralità di forme comunicative (all'interno della struttura catalogica del poema si intrecciano indistricabilmente e ambiguamente discours argumentatif e discours mythique), proietta dunque la narratività come cornice entro cui Parmenide avrebbe posto la stessa argomentazione e i suoi principi logici, insieme alla progressiva scoperta dell'Eon (p. 247). Come confermerebbe il frammento DK B2, con l'esplicito richiamo della Dea al proprio mythos (che nella nuova versione di Couloubaritsis suona «façon de parler autorisée») e soprattutto con la libertà di un chemin mythique che consente di delineare la via «qu'(il) n'est pas» per poi logicamente escludere immediatamente la possibilità di to me eon. Un nodo fondamentale non solo nella letteratura critica, ma anche nell'orizzonte della nuova interpretazione dell'autore, il quale coglie nella decisione (krisis) che taglia ouk esti la scelta (krisis) per esti, cioè per «le fait 
d'être comme tel», in altre parole per l'essere nel suo attuale irrompere nel presente: «fait d'être» e «être dans le présent» sarebbero la stessa cosa (p. 267).

$\grave{\mathrm{E}}$ in forza dell'instaurazione di «ciò che è nel presente» (eon), compreso nella sua temporalità limitata all'«ora», che «il pensare» (to noein) può costituirsi nella sua continuità e unità, e «essere rivelato» (pephatismenon), come Couloubaritsis interpreta B8.35-36: "perché senza "Ciò che è nel presente", grazie a cui è stato rivelato, tu non troverai il pensare». Traduzioni come «être»o «étant» avrebbero tradito con un anacronismo - che l'autore svela a partire dalla distinzione di Diogene di Apollonia tra eonta (appunto «cose che sono nel presente») e onta («cose che sono») - la specifica funzione di referenza ultima per ogni tipologia di discorso riflessivo e ogni forma logica che Parmenide attribuisce a eon. Ciò che risiede in se stesso, nella sua propria, irriducibile unità, in un «ora» permanente è anche il luogo da cui è possibile rivolgere lo sguardo alle cose in divenire, per costruirne un sapere conveniente: in virtù di una facoltà di pensare che fa emergere «cose presenti» (pareonta) a partire da «cose che non sono presenti» (me eonta) $\mathrm{o}$ «cose che sono assenti» (apeonta) (p. 394). In questo senso, come viene segnalato in prefazione, è il pensiero il vero oggetto della ricerca di Couloubaritsis: e Il pensiero di Parmenide e la questione del pensiero doveva esserne originariamente la titolazione.

I risultati dell'opera sono notevoli quanto a spunti di meditazione e provocazioni critiche, che investono temi (cultura arcaica, mito) in cui l'autore rivela magistrale competenza e acume, sebbene le soluzioni testuali adottate appaiano un po' forzate e non sempre convincenti sul terreno filologico (per esempio l'inserimento di DK B4 dopo B8.41). La ricchezza di implicazioni e la complessità dell'operazione culturale che Couloubaritsis riconosce al poema, inoltre, sembrano più rivolte a sfidare la stereotipia di approccio al pensiero arcaico da parte dei contemporanei che non a una plausibile ricostruzione della elaborazione che giace al fondo dei versi dell'Eleate. Nella misura in cui si tenga ben ferma la distinzione tra le potenzialità ermeneutiche che il poema dischiude e la concreta pratica di pensiero plausibilmente attribuibile a un autore vissuto e formatosi a cavaliere tra VI e V secolo a.C., la ricerca di Couloubaritsis si raccomanda per orientare il ricercatore tra le insidie delle facili riduzioni schematiche. Magari affiancata - per compensarne la sovrabbondanza di stimoli e di piste di indagine (e provocare lo sconcerto del lettore) - dalla unilaterale e apparentemente riduttiva lezione di In the Dark Places of Wisdom di P. Kingsley.

Dario ZUCCHELLO

François Roustang, Le Secret de Socrate pour changer la vie, Paris, Odile Jacob, 2009, 240 p., $€ 23,50$, ISBN 978-2-7381-2346-6

Socrate, on le sait, est une épine dans le pied de l'exégète, qui court toujours le risque de le banaliser ou - pire encore - de le remodeler à sa guise, jusqu'à en faire un Socrate «de complaisance ». Il est devenu difficile de produire un discours sérieux sur le compte d'un personnage aussi insaisissable. En effet, bien que les recherches se poursuivent à son sujet, nous nous trouvons depuis des décennies dans une situation persistante (et par là même fastidieuse) de stase vis- 